accuracy of UE (via trans-nasal or trans-oral insertion route) for the diagnosis of $\mathrm{BO}$ in adult patients with upper GI symptoms was analysed in this meta-analysis.

Methods A systematic literature search and meta-analysis was performed of all primary studies comparing UE (index test) to C-OGD. Electronic databases were searched from 1980 to November 1st2018 and non-English language articles were excluded. We excluded studies where data on accuracy or BO presence were not extractable or if they used technology obsolete in current practice (fibreoptic and prototype models).

Data extraction was undertaken by two reviewers independently of each other who then cross-checked the consistency of their findings. The quality of included studies was assessed using a validated tool (QUADAS-2). Heterogeneity was examined both by visual inspection of the Forest plots, and by statistical assessment using the chi square and inconsistency $\left(I^{2}\right)$ test. The bivariate model was used to obtain the estimates for accuracy. Data analysis was performed using Stata 12.1 software package (StataCorp, College Station, TX, USA).

Results Seven studies including 613 patients met the inclusion criteria. The pooled sensitivity, specificity, and area under the curve (AUC) for the diagnosis of $\mathrm{BO}$ were 0.98 (95\% confidence interval [CI] 0.84-1.00), 1.00 (95\% CI 0.86-1.00) and 1.00 (95\% CI $0.99-1.00)$ on a per-protocol analysis. There was significant heterogeneity between studies $\left(\mathrm{I}^{2}=0-95 \%\right.$; 95\% CI 0-100). Success rate for both UE and C-OGD was lowest at 0.86 in a small study using unsedated trans-oral route, while all the other studies reported success rates ranging from 0.89 to 1.0 for UE (including unsedated trans-nasal route) and from 0.98 to 1.0 for SE. The majority (0.54-0.71) of patients preferred UE compared to C-OGD and said they were willing to have UE again in the future if necessary. The mean tolerability scores for unsedated UE were significantly better in 4 studies, similar in 1 and not reported in the others.

Conclusions Unsedated UE is accurate in detecting BO and may be more acceptable to patients compared to C-OGD. However, there was significant heterogeneity across studies which limit the interpretation of the pooled results. This could have a bearing for future consideration of the value of Barrett's screening but its utility warrants further evaluation.

\section{PTH-044 EFFECT OF RENAL FAILURE ON THE OUTCOME OF UPPER GI HAEMORRHAGE INCLUDING RISK SCORING}

${ }^{1}$ Iain A Murray*, ${ }^{2}$ Rosie Haddock, ${ }^{3}$ Alisdair Menzies, ${ }^{4}$ David Fuchtbauer, ${ }^{4}$ Stig B Laursen, ${ }^{3}$ Nick Michell, ${ }^{2}$ Adrian I Stanley. ${ }^{1}$ Taunton and Somerset Foundation Trust, Taunton, UK; ${ }^{2}$ Glasgow Royal Infirmary, Glasgow, UK; ${ }^{3}$ Royal Cornwall Hospitals NHS Trust, Truro, UK; ${ }^{4}$ Odense University Hospital, Odense, Denmark

\subsection{6/gutjnl-2019-BSGAbstracts.69}

Introduction Renal failure is associated with poorer outcome following upper gastrointestinal haemorrhage (UGIH) and is a parameter in some pre-endoscopy risk stratification scores which can be used to identify low-risk patients who may avoid admission. We studied the outcome of patients admitted with UGIH in relation to their renal function and risk stratification scores.

Methods Prospectively collected data from a 1-year international multi-centre study of consecutive patients presenting with UGIH. Demographics, endoscopic findings, treatment and outcomes were recorded. eGFR was determined retrospectively from admission creatinine. If eGFR was $<60 \mathrm{ml} /$ minute, it was recorded whether this was acute kidney injury (AKI) or chronic kidney disease (CKD) by review of electronic patient notes. Glasgow Blatchford (GBS), admission Rockall (aRS), AIMS65 and PNED scores were determined. Need for endoscopic therapy, transfusion, surgery, rebleeding $>7$ days and 30-day mortality rates were recorded.

Results Mains findings are shown in table 1 as median or percentage except where indicated.

\begin{tabular}{|c|c|c|c|c|}
\hline & $\begin{array}{l}\text { Normal renal } \\
\text { function }\end{array}$ & AKI & CKD & $p$ value \\
\hline Number & 1208 & 165 & 250 & \\
\hline Age & 52 & 75 & 82 & $<0.0001$ \\
\hline Female & $39.7 \%$ & $42 \%$ & $50 \%$ & $<0.05$ \\
\hline Time to present (h) & 10 & 12 & 12 & NS \\
\hline Endoscopy findings: & $12.0 \%$ & $11.5 \%$ & $12.4 \%$ & NS \\
\hline Normal & $17.9 \%$ & $24.2 \%$ & $19.2 \%$ & \\
\hline Benign ulcer & $5.2 \%$ & $6.7 \%$ & $4.4 \%$ & \\
\hline Varices & $2.2 \%$ & $0 \%$ & $2.4 \%$ & \\
\hline Cancer & $27.6 \%$ & $24.2 \%$ & $18.4 \%$ & \\
\hline \multicolumn{5}{|l|}{ Not scoped } \\
\hline Endoscopic therapy & $18.6 \%$ & $25.9 \%$ & $27.6 \%$ & $<0.001$ \\
\hline Surgery & $0.6 \%$ & 0 & $1.2 \%$ & NS \\
\hline Rebleed $<7$ days & $4.6 \%$ & $8.4 \%$ & $6.8 \%$ & 0.06 \\
\hline 30-day mortality: & $1.2 \%$ & $7.2 \%$ & $4 \%$ & $<0.0001$ \\
\hline Bleed related & $3.1 \%$ & $10.8 \%$ & $13.2 \%$ & \\
\hline \multicolumn{5}{|l|}{ Non-bleed related } \\
\hline Transfusion & $27.7 \%$ & $41.6 \%$ & $49.6 \%$ & $<0.0001$ \\
\hline $\begin{array}{l}\text { Transfusion (average } \\
\text { units) }\end{array}$ & 3.7 & 4.7 & 4.4 & $<0.05$ \\
\hline $\begin{array}{l}\text { Need for treatment or } \\
\text { Died }\end{array}$ & $37.5 \%$ & $57.8 \%$ & $63.2 \%$ & $<0.0001$ \\
\hline $\begin{array}{l}\text { Length of stay (average } \\
\text { in days) }\end{array}$ & 3.96 & 8.11 & 6.58 & $<0.0001$ \\
\hline $\begin{array}{l}\text { GBS } \leq 1(\% \text {, sensitivity, } \\
\text { specificity) }\end{array}$ & $\begin{array}{l}30.0 \%, 94.6 \% \\
51.6 \%\end{array}$ & $\begin{array}{l}1.9 \%, 100 \% \\
58.9 \%\end{array}$ & $\begin{array}{l}2.0 \%, 100 \% \\
64.8 \%\end{array}$ & \\
\hline $\begin{array}{l}\text { AIMS65=0(\%,sensitivity, } \\
\text { specificity) }\end{array}$ & $\begin{array}{l}44.2 \%, 79.1 \%, \\
54.1 \%\end{array}$ & $\begin{array}{l}22.7 \%, 70.4 \%, \\
91.3 \%\end{array}$ & $\begin{array}{l}6.4 \%, 80 \% \\
66.8 \%\end{array}$ & \\
\hline $\begin{array}{l}\text { aRS=0 ( } \%, \text { sensitivity, } \\
\text { specificity) }\end{array}$ & $\begin{array}{l}21.7 \%, 85.6 \% \\
44.2 \%\end{array}$ & $\begin{array}{l}1.2 \%, 100 \% \\
58.8 \%\end{array}$ & $\begin{array}{l}1.6 \%, 75 \% \\
64.2 \%\end{array}$ & \\
\hline $\begin{array}{l}\text { PNED } \leq 1 \text { (\%, sensitivity, } \\
\text { specificity) }\end{array}$ & $\begin{array}{l}52.8 \%, 80.5 \%, \\
57.2 \%\end{array}$ & $\begin{array}{l}18.9 \%, 53.6 \% \\
63.3 \%\end{array}$ & $\begin{array}{l}12.4 \%, 67.9 \% \\
65.7 \%\end{array}$ & \\
\hline
\end{tabular}

Conclusions Patients admitted with UGIH and renal impairment are older and more likely to be female, than those with normal renal function. Transfusion requirements, need for endoscopic therapy, rebleed rate and mortality (bleed and non-bleed related) and are all increased in this group. GBS identifies low risk patients with AKI or CKD with high sensitivity, although few low risk patients are in this group. No other commonly used pre-endoscopy risk score is accurate in identifying low-risk patients with renal impairment. 